\title{
Accuracy of CT Angiography for Differentiating Pseudo-Occlusion from True Occlusion or High-Grade Stenosis of the Extracranial ICA in Acute Ischemic Stroke: A Retrospective MR CLEAN Substudy
}

\author{
(D) M. Kappelhof, (DH.A. Marquering, (D).A. Berkhemer, (D). Borst, (D)A. van der Lugt, (DW.H. van Zwam, (D).A. Vos, \\ (D) G. Lycklama à Nijeholt, DC.B.L.M. Majoie, and DB.J. Emmer, on behalf of the MR CLEAN Investigators
}

\begin{abstract}
BACKGROUND AND PURPOSE: The absence of opacification on CTA in the extracranial ICA in acute ischemic stroke may be caused by atherosclerotic occlusion, dissection, or pseudo-occlusion. The latter is explained by sluggish or stagnant flow in a patent artery caused by a distal intracranial occlusion. This study aimed to explore the accuracy of CTA for differentiating pseudo-occlusion from true occlusion of the extracranial ICA.

MATERIALS AND METHODS: All patients from the Multicenter Randomized Clinical Trial of Endovascular Treatment for Acute Ischemic Stroke in the Netherlands (MR CLEAN) with an apparent ICA occlusion on CTA and available DSA images were included. Two independent observers classified CTA images as atherosclerotic cause (occlusion/high-grade stenosis), dissection, or suspected pseudo-occlusion. Pseudo-occlusion was suspected if CTA showed a gradual contrast decline located above the level of the carotid bulb, especially in the presence of an occluded intracranial ICA bifurcation (T-occlusion). DSA images, classified into the same 3 categories, were used as the criterion standard.

RESULTS: In 108 of 476 patients (23\%), CTA showed an apparent extracranial carotid occlusion. DSA was available in 46 of these, showing an atherosclerotic cause in 13 (28\%), dissection in 16 (35\%), and pseudo-occlusion in $17(37 \%)$. The sensitivity for detecting pseudo-occlusion on CTA was $82 \%$ (95\% Cl, 57-96) for both observers; specificity was $76 \%(95 \% \mathrm{Cl}, 56-90)$ and $86 \%(95 \% \mathrm{Cl}, 68-96)$ for observers 1 and 2 , respectively. The $\kappa$ value for interobserver agreement was .77, indicating substantial agreement. T-occlusions were more frequent in pseudo- than true occlusions ( $82 \%$ versus $21 \%, P<.001)$.
\end{abstract}

CONCLUSIONS: On CTA, extracranial ICA pseudo-occlusions can be differentiated from true carotid occlusions.

ABBREVIATIONS: EVT = endovascular treatment; MR CLEAN = Multicenter Randomized Clinical Trial of Endovascular Treatment for Acute Ischemic Stroke in the Netherlands; T-occlusion $=$ occluded intracranial ICA bifurcation

$\mathbf{E}^{\mathrm{n}}$ ndovascular treatment (EVT) has been proved safe and effective in acute ischemic stroke caused by proximal intracranial occlusion if initiated within 6 hours of symptom onset. ${ }^{1}$ The duration and success of EVT are influenced by the ease of access to the intracranial vasculature. In approximately $25 \%$ of middle ce-

Received September 5, 2017; accepted after revision January 15, 2018.

From the Departments of Radiology (M.K., H.A.M., O.A.B., J.B., C.B.L.M.M, B.J.E.) and Biomedical Engineering and Physics (H.A.M.), Academic Medical Center, Amsterdam, the Netherlands; Departments of Radiology (A.v.d.L.) and Neurology (O.A.B.), Erasmus Medical Center, Rotterdam, the Netherlands; Department of Radiology (O.A.B., W.H.v.Z.), Maastricht University Medical Center, Maastricht, the Netherlands; Department of Radiology (J.A.V.), St. Antonius Hospital, Nieuwegein, the Netherlands; and Department of Radiology (G.L.à.N.), Haaglanden Medical Centre, The Hague, the Netherlands.

Paper previously presented at: European Stroke Organization Conference, May 10-12, 2016; Barcelona, Spain.

Please address correspondence to Bart J. Emmer, MD, PhD, Academic Medical Center, Department of Radiology, G1-240, Meibergdreef 9, 1105 AZ Amsterdam, the Netherlands; e-mail: b.j.emmer@amc.uva.nl

三 Indicates article with supplemental on-line table.

http://dx.doi.org/10.3174/ajnr.A5601 rebral artery strokes, concomitant intracranial artery and ipsilateral extracranial ICA occlusion occurs, known as tandem occlusion. $^{2}$ A recent study that included patients with tandem occlusions reported a median procedure time of 88 minutes $^{3}$ versus 30 and 43 minutes in 2 randomized trials that excluded patients with tandem occlusions. ${ }^{4,5}$

However, not all tandem occlusions identified on CTA represent true occlusions of the extracranial ICA. True occlusion of the extracranial ICA is caused by atherosclerosis or dissection of the vessel wall. So-called pseudo-occlusions mimic occlusion on CTA in the acute phase of ischemic stroke, while the artery is patent on DSA during EVT. ${ }^{6}$ The underlying mechanism of pseudo-occlusion of the extracranial ICA is sluggish or absent contrast flow caused by a distal occlusion of the intracranial vasculature. In these cases, CTA acquisition "outruns" the arrival of contrast material. ${ }^{6}$ Contrast flow can also be impeded by an extracranial ICA stenosis located proximal to the level of apparent occlusion. ${ }^{7,8}$

The term "pseudo-occlusion" has also been used for chronic high-grade stenosis of the ICA with minimal distal flow. ${ }^{9,10}$ This 
phenomenon is markedly different from pseudo-occlusion as an artifact of single-phase CTA, in which there is, in fact, no practically relevant carotid obstruction at all. ${ }^{6}$ To our knowledge, 6 previous studies have described a total of 48 patients with pseudoocclusions of the extracranial ICA in acute ischemic stroke. They reported that pseudo-occlusion occurs in 6\%-15\% of patients with acute ischemic stroke. ${ }^{6,11-15}$ It was stated that pseudo-occlusion cannot be discriminated from true occlusion on CTA. ${ }^{13}$ However, certain imaging characteristics may suggest pseudo-occlusion on CTA, such as an ipsilateral occluded intracranial ICA bifurcation (carotid T-occlusion) and good contrast filling of the carotid bulb followed by a gradual contrast decay in the ICA.

In the clinical setting, discrimination of pseudo-occlusion from true occlusion would improve estimation of the procedure time and intervention success by the operator. In case patients arrive late in their treatment window, time to overcome an extracranial ICA occlusion during treatment could be deemed too long for EVT to be beneficial to the patient. This could cause patients with unrecognized pseudo-occlusion on CTA to miss out on endovascular treatment that would have benefited them. Furthermore, better pseudo-occlusion recognition would enable more adequate planning and use of EVT materials such as guiding sheaths, percutaneous transluminal angioplasty balloons, and distal access catheters.

The aim of this retrospective study was to determine whether ICA pseudo-occlusion could be differentiated from atherosclerotic occlusion and dissection on CTA in the Multicenter Randomized Clinical Trial of Endovascular Treatment for Acute Ischemic Stroke in the Netherlands (MR CLEAN) dataset.

\section{MATERIALS AND METHODS}

This study was a blinded retrospective analysis of the data from the randomized controlled MR CLEAN trial. MR CLEAN included 500 patients with acute ischemic stroke caused by occlusion of an intracranial artery of the anterior circulation, with an NIHSS deficit of $\geq 2$ points, from 16 centers in the Netherlands. ${ }^{1,16}$ After arterial occlusion was demonstrated on vessel imaging, patients were randomized for usual care only or usual care with the addition of EVT. Treatment was initiated within $\leq 6$ hours from symptom onset. All imaging data were collected as part of MR CLEAN before the start of the current study.

\section{CTA Analysis}

Three raters (O.A.B., J.B., M.K.) assessed all baseline CTA scans for the presence of apparent ipsilateral occlusion of the extracranial ICA, without knowledge of angiographic findings. Images were reviewed in the axial, coronal, and sagittal planes. Only CTA images were assessed; noncontrast CT was not evaluated in this study. Minimum requirements for CTA scans in MR CLEAN were a slice thickness of $\leq 5 \mathrm{~mm}$, the presence of contrast in the intracranial vasculature, and depiction of at least the carotid bulb up to the circle of Willis. Median slice thickness was $0.9 \mathrm{~mm}$ (range, 0.5-2.0 mm), median scanner kiloelectron volt was 120 (range, 80-120), and the median number of scanner detector rows was 128 (range, 52-320). The total amount of injected contrast varied from 50 to $100 \mathrm{~mL}$ (median, $70 \mathrm{~mL}$ ) with a median injection speed of $5.0 \mathrm{~mL} / \mathrm{s}$ (range, 3.5-6.0 mL/s). Detailed CTA scan protocols per participating center can be found in the Online Table. "Apparent occlusion" was defined as the absence of contrast enhancement in the extracranial ICA ipsilateral to the intracranial occlusion.

Two experienced interventional and diagnostic neuroradiologists (B.J.E., G.L.à.N.), who were also blinded to the angiographic findings, separately reviewed scans with apparent occlusions. They categorized all cases into 1 of 3 underlying causes: atherosclerotic occlusion, dissection, or suspected pseudo-occlusion.

Atherosclerotic Occlusion. If an apparent occlusion was located at the level of the carotid bulb, was sharply demarcated, and/or showed a clear presence of calcifications or plaque, this apparent occlusion was considered as most likely caused by atherosclerosis and classified as such. ${ }^{17}$

Dissection. If an apparent ICA occlusion was located above the level of the carotid bulb, without plaque or calcifications at the carotid bulb, and the occlusion was sharply demarcated on CTA, the occlusion was classified as most likely caused by a dissection. Furthermore, young patient age and widening of the ICA under the skull base could suggest the presence of a dissection.

Suspected Pseudo-Occlusion. Pseudo-occlusion was suspected if CTA showed a gradual contrast decay in the ICA above the level of the carotid bulb, in the absence of plaque or calcifications around the carotid bulb, and in the presence of a carotid-T or large M1-occlusion.

Carotid T-Occlusion. T-occlusion presence was determined to compare the prevalence in pseudo- versus true occlusion cases. If a carotid top or terminus was not filled with contrast on CTA, it was classified as a carotid T-occlusion. All cases with apparent extracranial ICA occlusion on CTA and available DSA imaging were reviewed.

\section{DSA Analysis}

DSA images of cases with apparent occlusion on CTA were collected. To prevent recall bias, we gave the DSA images new patient identification numbers and rated them 6 months later than the CTAs. One rater (B.J.E.) reviewed all DSA images, checking for atherosclerotic occlusion or dissection. Contrast was injected in the common carotid artery and directly into the ICA in all cases. Dissection was confirmed in case of an irregular vessel wall (at the beginning of the procedure, before vasospasm could have arisen) with a tapering aspect of contrast leading up to an occlusion above the level of the carotid bulb that could not be passed with the guidewire or catheter. Atherosclerotic occlusion was confirmed in case of an abrupt, blunt contrast cutoff at the level of (or slightly above) the carotid bulb, with evidence of circumferential vessel wall involvement that could not be passed easily with a guidewire or catheter. If, despite an apparent occlusion on CTA, DSA showed only high-grade (90\%-99\%) atherosclerotic stenosis, the underlying reason for the apparent occlusion was deemed an atherosclerotic cause because the practical implications of atherosclerotic high-grade stenosis for EVT are similar to those of atherosclerotic occlusion. If DSA showed no occlusion, a low-grade stenosis, or an apparent occlusion that could be passed unhin- 
dered with a guidewire or catheter, the apparent ICA occlusion on CTA was deemed a pseudo-occlusion.

\section{Statistical Analysis}

SPSS, Version 24 (IBM, Armonk, New York) and R, Version 3.2 (http://www.r-project.org) were used for the statistical analysis. Sensitivity, specificity, and positive and negative predictive values were calculated for detection of pseudo-occlusions, atherosclerotic occlusions, and dissections on CTA. DSA images were used as the criterion standard. Ninety-five percent confidence intervals were calculated using the Clopper-Pearson method for sensitivity and specificity and the asymptotic/adjusted logit intervals for predictive values. Interobserver variability of CTA-based pseudo-occlusion recognition was tested with the $\kappa$ statistic and the Krippendorff $\alpha$, to control for the factor of random agreement. The Fisher exact test was used to calculate the statistical significance of the difference in T-occlusion rates in subgroups with and without pseudo-occlusions.

\section{RESULTS}

\section{Patients}

Figure 1 depicts patient inclusion. CTA images were available for 476 patients. The intervention arm of MR CLEAN com-

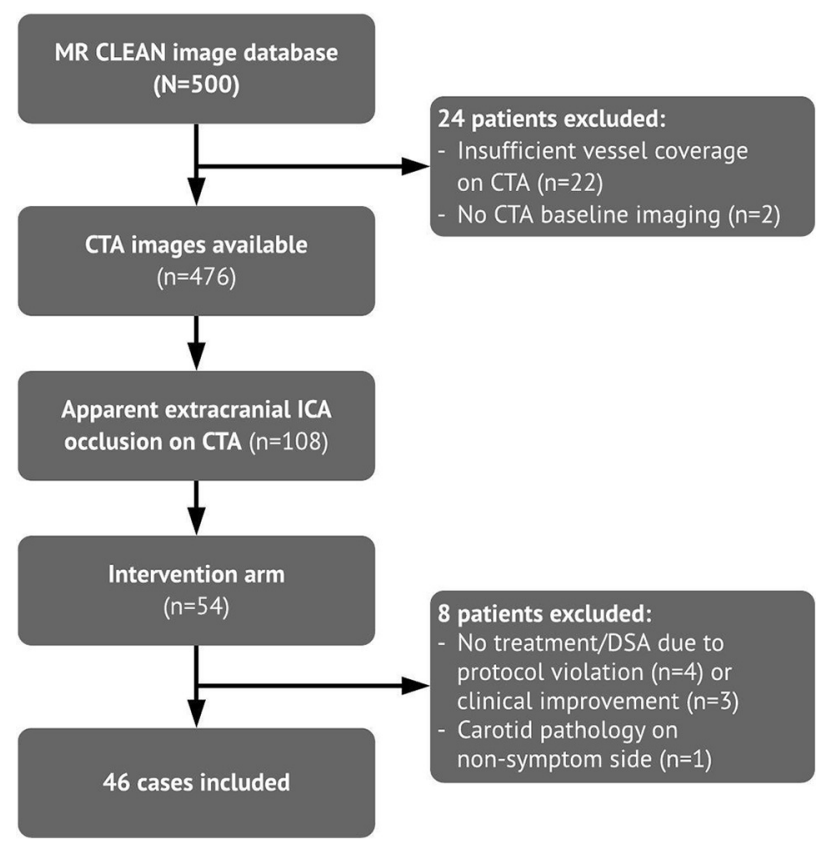

FIG 1. Patient-selection flow chart. $n$ indicates number of cases. prised 233 patients, of whom 216 had DSA images available for analysis. ${ }^{1}$ Apparent extracranial ICA occlusion on CTA, regardless of the cause, was found in 108 patients. Forty-six of them had DSA images available and thus were included in the current study.

\section{CTA Analysis}

Pseudo-occlusion was identified in 14/17 (82\%) cases by both observers (Table 1). For observer 1, the sensitivity and specificity of CTA for identifying pseudo-occlusion were 82\% (95\% CI, 57\%-96\%) and 76\% (95\% CI, 56\%-90\%). For observer 2, these numbers were $82 \%(95 \% \mathrm{CI}, 57 \%-96 \%)$ and $86 \%(95 \%$ CI, 68\%-96\%), respectively (Table 2). The $\kappa$ value for interobserver agreement on pseudo-occlusions was .77 (95\% CI, $0.56-0.97)$. The Krippendorf $\alpha$ for interobserver agreement on pseudo-occlusions, atherosclerotic occlusions, and dissections was .77 (95\% CI, .61-.90).

Dissection and pseudo-occlusion were most frequently not distinguished correctly from each other on CTA. For observers 1 and 2, respectively, 29\% (6/21) and 22\% (4/18) of suspected pseudo-occlusions turned out to be dissections. Conversely, 23\% (3/13) and 20\% (3/15) of CTA-diagnosed dissections turned out to be pseudo-occlusions (Table 1). Atherosclerotic pathologies were well-recognized, with a sensitivity of $92 \%(95 \% \mathrm{CI}, 64 \%-100 \%)$ and $100 \%$ (95\% CI, $75 \%-100 \%$ ) for observers 1 and 2 , respectively (Table 2 ).

Figure 2 shows examples of cases with matching classification of atherosclerotic occlusion, dissection, and pseudo-occlusion, respectively, on CTA and DSA. Figure 3 depicts examples of the most common misinterpretations: cases in which CTA suggested pseudo-occlusion, whereas DSA showed dissection, or CTA suggested dissection, whereas DSA showed pseudo-occlusion. An apparent occlusion located above the level of the carotid bulb, in the absence of extensive amounts of calcifications or plaque, is present in both dissections and pseudo-occlusions, making distinction of the diagnoses more difficult. However, dissections often show a less gradual decline of contrast in the ICA, more tapering of the vessel, and sometimes widening of the ICA contour under the skull base. Furthermore, the presence of carotid T-occlusion may contribute to identification of the right diagnosis.

\section{T-Occlusions}

T-occlusions were present in 14 of 17 (82\%) pseudo-occlusions versus 2 of $13(15 \%)$ atherosclerotic cases and 4 of $16(25 \%)$ dis-

Table 1: Outcome of diagnoses on CTA for observers 1 and 2 versus final diagnosis on DSA

\begin{tabular}{|c|c|c|c|c|c|}
\hline \multirow[b]{3}{*}{ CTA } & \multicolumn{4}{|c|}{ DSA (No.) } & \multirow[b]{3}{*}{ Total No. } \\
\hline & \multicolumn{2}{|c|}{ Atherosclerotic Cause } & \multirow[b]{2}{*}{ Pseudo-Occlusion } & \multirow[b]{2}{*}{ Dissection } & \\
\hline & Occlusion & High-Grade Stenosis & & & \\
\hline \multicolumn{6}{|l|}{ Observer 1} \\
\hline Atherosclerotic occlusion & 10 & 2 & 0 & 0 & 12 \\
\hline Pseudo-occlusion & 0 & 1 & 14 & 6 & 21 \\
\hline Dissection & 0 & 0 & 3 & 10 & 13 \\
\hline Total & & 13 & 17 & 16 & 46 \\
\hline \multicolumn{6}{|l|}{ Observer 2} \\
\hline Atherosclerotic occlusion & 10 & 3 & 0 & 0 & 13 \\
\hline Pseudo-occlusion & 0 & 0 & 14 & 4 & 18 \\
\hline Dissection & 0 & 0 & 3 & 12 & 15 \\
\hline Total & & 13 & 17 & 16 & 46 \\
\hline
\end{tabular}


sections $(P<.001)$. Of all carotid T-occlusions, 70\% (14/20) showed concomitant pseudo-occlusion.

T-occlusions were correctly recognized on CTA in all cases except 1 . In that case, CTA showed a floating thrombus in the carotid siphon, which had possibly embolized into the carotid-T by the time DSA images were acquired. Two pseudo-occlusion cases did not show a carotid T-occlusion on DSA. In one, carotid T-occlusion was seen on CTA, and DSA showed an M1 occlusion.

\section{Table 2: Diagnostic accuracy measures for pseudo-occlusion recognition on CTA compared} with DSA for observers 1 and 2

\begin{tabular}{lccc}
\hline & Pseudo-Occlusion & $\begin{array}{c}\text { Atherosclerotic } \\
\text { Cause }\end{array}$ & Dissection \\
\hline Observer 1 & & & \\
Sensitivity (95\% Cl) & $82(57-96)$ & $100(64-100)$ & $63(35-85)$ \\
Specificity (95\% CI) & $76(56-90)$ & $100(76-100)$ & $90(73-98)$ \\
PPV & $67(50-80)$ & $97(83-100)$ & $77(52-92)$ \\
NPV & $88(72-95)$ & & $82(70-90)$ \\
Observer 2 & & $100(75-100)$ & $75(47-93)$ \\
Sensitivity (95\% CI) & $82(57-96)$ & $100(89-100)$ & $90(73-98)$ \\
Specificity (95\% Cl) & $86(68-96)$ & $100(77-100)$ & $80(57-92)$ \\
PPV & $78(58-90)$ & $100(90-100)$ & $87(74-94)$ \\
NPV & $89(75-95)$ &
\end{tabular}

The other case showed M1 and A1 occlusion on CTA, and M2 occlusion on DSA. Migration of the thrombus, possibly under the influence of IV tPA, could explain these cases.

\section{DSA Analysis}

The prevalence of pseudo-occlusions in all patients in MR CLEAN with DSA imaging available was $8 \%$ (17 of 216 patients). Of 46 included cases with apparent occlusion on CTA, DSA showed atherosclerotic occlusion or high-grade stenosis in 13 cases $(28 \%$, of which 3 [7\% in total] were high-grade stenoses) and dissection in $16(35 \%)$. In 17 patients, no carotid occlusion or high-grade stenosis was found; therefore, $37 \%$ of apparent extracranial ICA occlusions on CTA were pseudo-occlusions (Table 1). These cases usually showed delayed or absent contrast filling of the vessel distal to the carotid bulb with an indistinct or oscillating contrast border on DSA.

Note:-PPV indicates positive predictive value; NPV, negative predictive value.

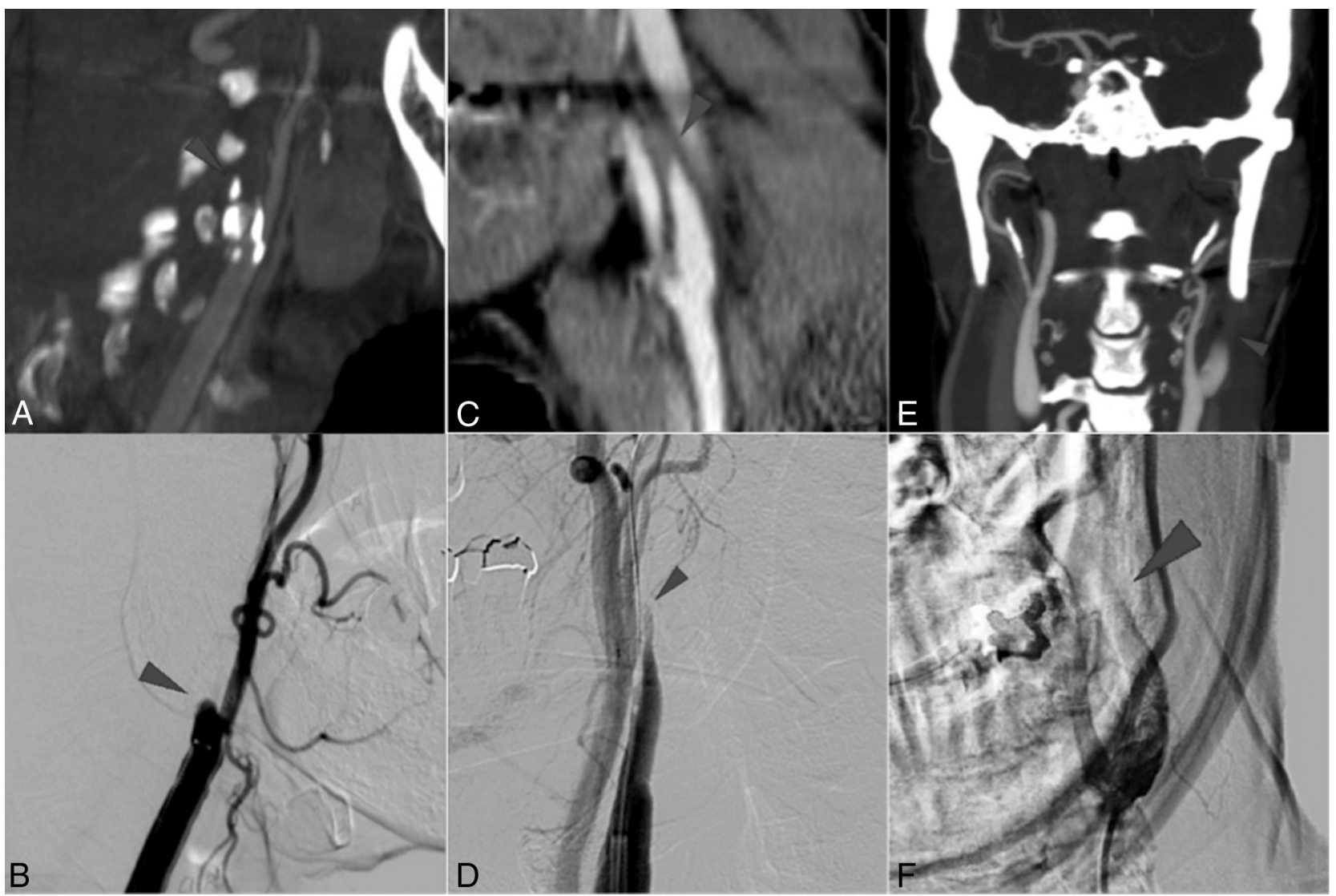

FIG 2. Examples of carotid pathologies on CTA (upper row) and corresponding DSA (lower row). A, Atherosclerotic occlusion of the right ICA in a 79-year-old man. CTA shows extensive calcifications in the carotid bulb with a sharp contrast cutoff at the level of the bifurcation. $B$, DSA of the patient in A shows a sharp delineation of contrast at the level of the carotid bulb, and "shouldering," a convex aspect of the contrast cutoff proximal to the occlusion. C, Dissection of the left ICA in a 44-year-old man. CTA shows a sharp, diagonal contrast cutoff above the level of the carotid bifurcation. D, DSA of the patient in C shows a gradual decline in vessel diameter also known as "tapering," with a sharp contrast cutoff and irregular vessel wall. It was possible to pass the apparent occlusion with a guidewire, but not with a catheter. E, Pseudo-occlusion of the left ICA in a 51-year-old woman. CTA shows a gradual contrast decrease in the left ICA above the level of the carotid bulb (arrowhead) compared with a normal contrast density on the contralateral side, and an ipsilateral carotid T-occlusion. $F$, DSA of the patient in $E$ shows a blurred contrast delineation above the level of the carotid bulb. A back-and-forth moving, "oscillating," contrast border was seen. The apparent occlusion could easily be passed with a guidewire or catheter. 


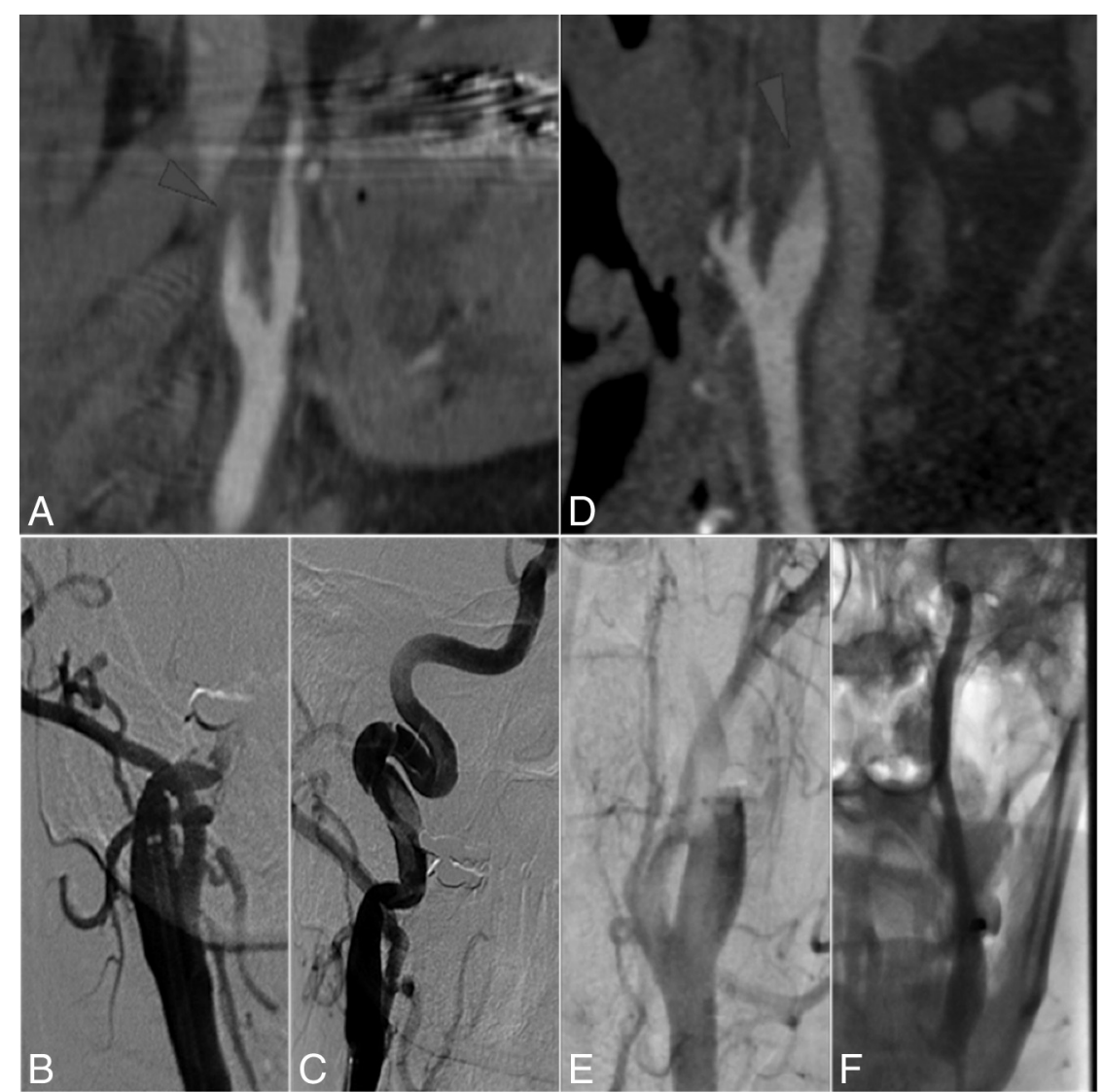

FIG 3. Examples of suspected dissection or pseudo-occlusion on CTA with a noncorresponding cause of apparent occlusion found on DSA. A, Dissection of the right ICA in a 65-year-old woman. CTA shows a blurred contrast cutoff above the level of the carotid bulb, suggesting pseudoocclusion, without T-occlusion present. $B$, DSA of the patient in $A$ shows major vessel wall irregularities and the impossibility of passing the occlusion with a catheter $(C)$, indicating the presence of a dissection rather than pseudo-occlusion. $D$, Pseudo-occlusion of the left ICA in a 63-year-old man. CTA shows a sharp, diagonal contrast cutoff above the level of the carotid bifurcation, suggesting dissection, with T-occlusion present. E, DSA of patient in D shows a blurred contrast cutoff slowly moving upward and finally a patent ICA with intracranial carotid T-occlusion present $(F)$, indicating pseudo-occlusion.

\section{DISCUSSION}

The main finding of this study is that on CTA made in preparation for EVT, extracranial ICA pseudo-occlusion can be well-differentiated from atherosclerotic occlusion and reasonably well from carotid dissection. Additionally, in about one-third of patients with an apparent extracranial internal carotid occlusion on CTA in acute ischemic stroke, the artery is, in fact, patent on DSA during EVT. Finally, the prevalence of a carotid T-occlusion in patients with carotid pseudo-occlusion was high.

To our knowledge, there are no reports on the diagnostic accuracy of CTA on the etiology of apparent tandem lesions in acute stroke with proximal large-vessel occlusions. Previous research $^{6,11-13}$ reported the prevalence of pseudo-occlusions to be $6 \%-14 \%$. However, these studies included cases in which highgrade extracranial ICA stenosis impeded contrast flow, and they considered them pseudo-occlusions as well. ${ }^{6,11-13}$ Furthermore, previous studies found a high prevalence of carotid T-occlusions in pseudo-occlusion cases. ${ }^{11-13}$

We have introduced a CTA-based diagnostic approach that could differentiate pseudo-occlusion from true occlusion or high-grade atherosclerotic stenosis with high sensitivity and specificity. Imaging characteristics suggestive of pseudo-occlusions were the presence of a carotid Tocclusion and a gradual contrast decline located above the level of the carotid bulb in the absence of atherosclerotic plaque or calcifications. The prevalence of pseudoocclusions in MR CLEAN was in line with the findings in the studies above. Likewise, in our study, in most pseudo-occlusions, a carotid T-occlusion was present. In a minority of our patients, DSA showed highgrade atherosclerotic stenosis proximal to a gradual contrast decay in the ICA, similar to cases described before. ${ }^{6,11,12}$ However, because high-grade atherosclerotic stenoses usually require a similar approach to atherosclerotic occlusions during EVT, in acute ischemic stroke, one could argue that these cases should be classified as atherosclerotic occlusions.

Several limitations to this study should be noted. First, we have no data on occlusion patterns and characteristics of patients who were not randomized. A selection bias might have occurred because local investigators might have opted not to include patients with extracranial ICA occlusions. Although this might have influenced the prevalence of apparent tandem occlusions in the study sample, it would not have affected the reported sensitivity and specificity measures. Besides, compared with other randomized controlled trials on $\mathrm{EVT},{ }^{4,5,18,19}$ the number of patients with tandem occlusions included in MR CLEAN is relatively high.

Second, the initial selection of apparent ICA occlusions from the dataset was made by 3 researchers who were, though experienced, not certified radiologists. Carotid occlusions might have been missed. However, again this approach would not have affected the sensitivity and specificity numbers because apparent occlusion causes were determined by experienced interventional neuroradiologists.

Finally, this study showed the distinction between carotid dissections and pseudo-occlusions to be the most difficult to make. Both showed a contrast decline above the level of the carotid bifurcation in the absence of carotid plaque or calcifications. A possible discriminating characteristic is a sharp contrast cutoff in dissections versus a gradual contrast decline in pseudo-occlusions. This gradual contrast decline was previously described as the "midcervical flame-shaped extracranial ICA sign." ${ }^{14}$ However, contrast border appearance can be affected by bolus timing, scan acquisition, and low-grade proximal ICA stenosis. These factors may have influenced observers' decisions on apparent occlusion classification and thereby sensitivity and specificity measures. 
CTA scan timing may influence the occurrence and recognition of carotid pseudo-occlusion. Late-phase CTA scans may show a patent ICA by allowing slow contrast flow in the ICA to reach the actual intracranial occlusion. Determining optimal scanning conditions for pseudo-occlusion differentiation on single-phase CTA would require further research. Acquiring a second, late-phase scan after the initial CTA may be of additional value.

Other imaging modalities used in the acute setting of stroke may improve recognition of pseudo-occlusions. For example, 4D-CTA was recently shown to successfully identify slow flow causing pseudo-occlusions. ${ }^{13,20,21}$

\section{CONCLUSIONS}

Extracranial ICA pseudo-occlusion can be well-differentiated from atherosclerotic occlusion and reasonably well from carotid dissection on CTA. The prevalence of pseudo-occlusion in the MR CLEAN trial was high. Several occlusion characteristics can raise suspicion for pseudo-occlusion on CTA, most importantly concomitant carotid T-occlusion.

\section{ACKNOWLEDGMENTS}

MR CLEAN trial investigators (all from the Netherlands unless stated otherwise): Peter J. Koudstaal, MD, PhD, and Diederik W.J. Dippel, MD, PhD, Department of Neurology, Erasmus MC University Medical Center, Rotterdam; Yvo B.W.E.M. Roos, MD, $\mathrm{PhD}$, Lucie A. van den Berg, MD, and Paul J. Nederkoorn, MD, PhD, Department of Neurology, Academic Medical Center, Amsterdam; Robert J. van Oostenbrugge, $\mathrm{MD}$, PhD, Debbie Beumer, $\mathrm{MD}$, and Julie Staals, MD, PhD, Department of Neurology, Maastricht University Medical Center, Cardiovascular Research Institute Maastricht; Jelis Boiten, MD, PhD, Department of Neurology, Medisch Centrum Haaglanden, The Hague; Marieke J.H. Wermer, MD, PhD, Department of Neurology, Leiden University Medical Center; L. Jaap Kappelle, MD, PhD, Department of Neurology, University Medical Center Utrecht; Ewoud J. van Dijk, $\mathrm{MD}, \mathrm{PhD}$, Department of Neurology, Radboud University Medical Center, Nijmegen; Wouter J. Schonewille, MD, PhD, Department of Neurology, St. Antonius Hospital, Nieuwegein; Willem Jan van Rooij, MD, PhD, Department of Radiology, St. Elisabeth Hospital, Tilburg; Jeannette Hofmeijer, MD, PhD, and Jacques A. van Oostayen, $\mathrm{MD}, \mathrm{PhD}$, Department of Radiology, Rijnstate Hospital, Arnhem; Patrick C. Vroomen, MD, PhD, Department of Neurology, University Medical Center Groningen; Paul L.M. de Kort, MD, PhD, Department of Neurology, St. Elisabeth Hospital, Tilburg; Koos Keizer, MD, PhD, Department of Neurology, Catharina Hospital, Eindhoven; Sebastiaan F. de Bruijn, MD, $\mathrm{PhD}$, Department of Neurology, Haga Hospital, The Hague; J.S. Peter van den Berg, $\mathrm{MD}, \mathrm{PhD}$, Department of Neurology, Isala Klinieken, Zwolle; Tobien H.C.M.L. Schreuder, MD, Department of Neurology, Atrium Medical Center, Heerlen; Leo A.M. Aerden, $\mathrm{MD}, \mathrm{PhD}$, and H. Zwenneke Flach, MD, Department of Neurology, Reinier de Graaf Gasthuis, Delft; Marieke C. Visser, MD, $\mathrm{PhD}$, Department of Neurology, VU Medical Center, Amsterdam; Heleen M. den Hertog, MD, PhD, Department of Neurology, Medical Spectrum Twente, Enschede; Jan S.P. van den Berg, $\mathrm{MD}, \mathrm{PhD}$, Department of Neurology, Isala Klinieken, Zwolle;
Patrick A. Brouwer, MD, Department of Radiology, Leiden University Medical Center; Aad van der Lugt, MD, PhD, Department of Radiology, Erasmus MC University Medical Center, Rotterdam; Bart J. Emmer, MD, PhD, Charles B.L.M. Majoie, MD, PhD, Ludo F.M. Beenen, MD, Marieke E.S. Sprengers, MD, PhD, René van den Berg, $\mathrm{MD}, \mathrm{PhD}$, and Olvert A. Berkhemer, $\mathrm{MD}$, Department of Radiology, Academic Medical Center, Amsterdam; Wim H. van Zwam, MD, PhD, Department of Radiology, Maastricht University Medical Center; Geert J. Lycklama à Nijeholt, MD, $\mathrm{PhD}$, Department of Radiology, Medisch Centrum Haaglanden, The Hague; Marianne A.A. van Walderveen, MD, PhD, Department of Radiology, Leiden University Medical Center; Rob H. Lo, MD, Department of Radiology, University Medical Center Utrecht; Joost de Vries, MD, PhD, Department of Neurosurgery, Radboud University Medical Center, Nijmegen; Jan Albert Vos, $\mathrm{MD}, \mathrm{PhD}$, Department of Radiology, St. Antonius Hospital, Nieuwegein; Omid Eshghi, MD, Department of Radiology, University Medical Center, Groningen; Alexander V. Tielbeek, MD, $\mathrm{PhD}$, Department of Radiology, Catharina Hospital, Eindhoven; Lukas C. van Dijk, MD, Department of Radiology, Haga Hospital, The Hague; Boudewijn A.A.M. van Hasselt, MD, Department of Radiology, Isala Klinieken, Zwolle; Roel J.J. Heijboer, MD, Department of Radiology, Atrium Medical Center, Heerlen; René J. Dallinga, MD, Department of Radiology, Reinier de Graaf Gasthuis, Delft; Joseph C.J. Bot, MD, PhD, Department of Radiology, VU Medical Center, Amsterdam; Dick G. Gerrits, MD, Department of Radiology, Medical Spectrum Twente, Enschede; Puck S.S. Fransen, MD, Departments of Neurology and Radiology, Erasmus MC University Medical Center, Rotterdam; Henk A. Marquering, $\mathrm{PhD}$, Departments of Radiology and Biomedical Engineering and Physics, Academic Medical Center, Amsterdam; Hester F. Lingsma, PhD, and Ewout W. Steyerberg, PhD, Department of Public Health, Erasmus MC University Medical Center, Rotterdam; Albert J. Yoo, MD, Texas Stroke Institute, Plano, Texas; Sjoerd F.M. Jenniskens, MD, PhD, Department of Radiology, Radboud University Medical Center, Nijmegen; Renske M. van den Berg-Vos, MD, PhD, Department of Neurology, St. Lucas Andreas Hospital, Amsterdam; and Giorgos B. Karas, MD, PhD, MBA, Department of Radiology, St. Lucas Andreas Hospital, Amsterdam. The data monitoring and safety board included Martin M. Brown, MD (chair), National Hospital for Neurology and Neurosurgery, London, United Kingdom; Thomas Liebig, MD, Medizinische Fakultät, Universität zu Köln, Germany; and Theo Stijnen, PhD, Leiden University Medical Center (independent statistician). The advisory board included Tommy Andersson, MD (neurointerventionist), Karolinska University Hospital, Stockholm, Sweden; Heinrich Mattle, MD (neurologist), University Hospital, Bern, Switzerland; and Nils Wahlgren, MD (neurologist), Karolinska University Hospital, Stockholm, Sweden. Research nurses and local trial coordinators included Esther van der Heijden and Naziha Ghannouti, Erasmus MC University Medical Center, Rotterdam; Nadine Fleitour and Imke Hooijenga, Academic Medical Center, Amsterdam; Corina Puppels and Wilma Pellikaan, St. Antonius Hospital, Nieuwegein; Annet Geerling, Radboud University Medical Center, Nijmegen; Annemieke Lindl-Velema, Maastricht University Medical Center; Gina van Vemde and Isala Klinieken, Zwolle; Ans de Ridder and Paut 
Greebe, University Medical Center, Utrecht; José de Bont-Stikkelbroeck, St Elisabeth Hospital, Tilburg; Joke de Meris, Medisch Centrum Haaglanden, The Hague; Kirsten Janssen, Leiden University Medical Center; Willy Struijk, Haga Hospital, The Hague; and Silvan Licher, Nikki Boodt, Adriaan Ros, Esmee Venema, Ilse Slokkers, Raymie-Jayce Ganpat, Maxim Mulder, Nawid Saiedie, Alis Heshmatollah, Stefanie Schipperen, Stefan Vinken, Tiemen van Boxtel, and Jeroen Koets, Erasmus MC University Medical Center, Rotterdam.

Disclosures: Henk A. Marquering—UNRELATED: Stock/Stock Options: Nico.lab, Comments: founder and shareholder. Olvert A. Berkhemer-UNRELATED: Consultancy: Stryker.* Aad van der Lugt—UNRELATED: Consultancy: Stryker.* Wim H. van Zwam-UNRELATED: Consulting Fee or Honorarium: Stryker, Codman Neuro.* Charles B.L.M. Majoie-UNRELATED: Consultancy: Stryker*; Grants/Grants Pending: TWIN Foundation, CVON/Dutch Heart Foundation. * Bart J. Emmer-UNRELATED: Consultancy: Stryker*; Expert Testimony: DEKRA Certification B.V., Comments: CE Mark review fees; Payment for Lectures Including Service on Speakers Bureaus: Novartis. *Money paid to the institution.

\section{REFERENCES}

1. Berkhemer OA, Fransen PS, Beumer D, et al. A randomized trial of intraarterial treatment for acute ischemic stroke. N Engl J Med 2015; 372:11-20 CrossRef Medline

2. Dababneh H, Bashir A, Hussain M, et al. Endovascular treatment of tandem internal carotid and middle cerebral artery occlusions. J Vasc Interv Neurol 2014;14:25-30 Medline

3. Behme D, Mpotsaris A, Zeyen P, et al. Emergency stenting of the extracranial internal carotid artery in combination with anterior circulation thrombectomy in acute ischemic stroke: a retrospective multicenter study. AJNR Am J Neuroradiol 2015;36:2340-45 CrossRef Medline

4. Campbell BC, Mitchell PJ, Kleinig TJ, et al; EXTEND-IA Investigators. Endovascular therapy for ischemic stroke with perfusion-imaging selection. $N$ Engl J Med 2015;372:1009-18 CrossRef Medline

5. Saver JL, Goyal M, Bonafe A, et al; SWIFT PRIME Investigators. Stent-retriever thrombectomy after intravenous t-PA vs. t-PA alone in stroke. $N$ Engl J Med 2015;372:2285-95 CrossRef Medline

6. Kim JJ, Dillon WP, Glastonbury CM, et al. Sixty-four-section multidetector CT angiography of carotid arteries: a systematic analysis of image quality and artifacts. AJNR Am J Neuroradiol 2010;31:91-99 CrossRef Medline

7. Korn A, Bender B, Brodoefel H, et al. Grading of carotid artery stenosis in the presence of extensive calcifications: dual-energy CT angiography in comparison with contrast-enhanced MR angiography. Clin Neuroradiol 2015;25:33-40 CrossRef Medline

8. Fujimoto S, Toyoda K, Kishikawa K, et al. Accuracy of conventional plus transoral carotid ultrasonography in distinguishing pseudo- occlusion from total occlusion of the internal carotid artery. Cerebrovasc Dis 2006;22:170-76 CrossRef Medline

9. Fürst G, Saleh A, Wenserski F, et al. Reliability and validity of noninvasive imaging of internal carotid artery pseudo-occlusion. Stroke 1999;30:1444-49 CrossRef Medline

10. Desole A, Campanile F, Tosato F, et al. Surgical treatment for pseudo-occlusion of the internal carotid artery. Interact Cardiovasc Thorac Surg 2015;20:636-40 CrossRef Medline

11. Marquering HA, Nederkoorn PJ, Beenen LF, et al. Carotid pseudoocclusion on CTA in patients with acute ischemic stroke: a concerning observation. Clin Neurol Neurosurg 2013;115:1591-94 CrossRef Medline

12. Duijsens HM, Spaander F, van Dijk LC, et al. Endovascular treatment in patients with acute ischemic stroke and apparent occlusion of the extracranial internal carotid artery on CTA. J Neurointerv Surg 2015;7:709-14 CrossRef Medline

13. Ng FC, Choi PM, Datta M, et al. Perfusion-derived dynamic 4D CT angiography identifies carotid pseudo-occlusion in hyperacute stroke. J Neuroimaging 2016;26:588-91 CrossRef Medline

14. Prakkamakul S, Pitakvej N, Dumrongpisutikul N, et al. Mid-cervical flame-shaped pseudo-occlusion: diagnostic performance of midcervical flame-shaped extracranial internal carotid artery sign on computed tomographic angiography in hyperacute ischemic stroke. Neuroradiology 2017;59:989-96 CrossRef Medline

15. Akpınar S, Gelener P, Yilmaz G. Aetiologies of internal carotid artery pseudo-occlusions in acute stroke patients: what neurointerventionalists can expect. Br J Radiol 2017;90:20160352 CrossRef Medline

16. Fransen PS, Beumer D, Berkhemer OA, et al; MR CLEAN Investigators. MR CLEAN, a multicenter randomized clinical trial of endovascular treatment for acute ischemic stroke in the Netherlands: study protocol for a randomized controlled trial. Trials 2014;15:343 CrossRef Medline

17. Park ST, Kim JK, Yoon KH, et al. Atherosclerotic carotid stenoses of apical versus body lesions in high-risk carotid stenting patients. AJNR Am J Neuroradiol 2010;31:1106-12 CrossRef Medline

18. Goyal M, Demchuk AM, Menon BK, et al; ESCAPE Trial Investigators. Randomized assessment of rapid endovascular treatment of ischemic stroke. N Engl J Med 2015;372:1019-30 CrossRef Medline

19. Jovin TG, Chamorro A, Cobo E, et al; REVASCAT Trial Investigators. Thrombectomy within $\mathbf{8}$ hours after symptom onset in ischemic stroke. N Engl J Med 2015;372:1-11 CrossRef Medline

20. Miyazaki Y, Mori T, Tajiri H, et al. Comparison of four-dimensional CT angiography (4D-CTA) with MRA in acute ischemic stroke patients with probable internal carotid artery occlusion. Stroke 2012;43:A2859

21. Zhang S, Chen W, Tang H, et al. The prognostic value of a fourdimensional CT angiography-based collateral grading scale for reperfusion therapy in acute ischemic stroke patients. PLoS One 2016;11:1-12 CrossRef Medline 